\title{
Some Properties of the Class of Univalent Functions with Negative Coefficients
}

\author{
Aisha Ahmed Amer, Maslina Darus* \\ School of Mathematical Sciences, Faculty of Science and Technology, \\ Universiti Kebangsaan Malaysia, Bangi, Malaysia \\ Email: eamer_80@yahoo.com, ${ }^{*}$ maslina@ukm.my
}

Received August 6, 2012; revised October 17, 2012; accepted October 26, 2012

\begin{abstract}
The main object of this paper is to study some properties of certain subclass of analytic functions with negative coefficients defined by a linear operator in the open unit disc. These properties include the coefficient estimates, closure properties, distortion theorems and integral operators.
\end{abstract}

Keywords: Analytic Function; Unit Disc; Coefficient Inequality; Closure Properties; Distortion Bound

\section{Introduction}

Let $\mathcal{H}$ be the class of analytic functions in the open unit disc

$$
U=\{z \in \mathbb{C}:|z|<1\},
$$

and $\mathcal{H}[a, n]$ be the subclass of $\mathcal{H}$ consisting of functions of the form

$$
f(z)=a+a_{n} z^{n}+a_{n+1} z^{n+1}+\cdots .
$$

Let $\mathcal{A}(n)$ denote the class of functions $f(z)$ normalized by

$$
f(z)=z+\sum_{k=n+1}^{\infty} a_{k} z^{k},(n \in \mathbb{N}:=\{1,2,3, \cdots\}),
$$

which are analytic in the open unit disc. In particular,

$$
\mathcal{A}(1):=\mathcal{A} \text {. }
$$

For two functions $f(z)$ given by (1) and $g(z)$ given by

$$
g(z)=z+\sum_{k=n+1}^{\infty} b_{k} z^{k},(n \in \mathbb{N}),
$$

the Hadamard product (or convolution) $(f * g)(z)$ is defined, as usual, by

$$
(f * g)(z):=z+\sum_{k=n+1}^{\infty} a_{k} b_{k} z^{k}:=(g * f)(z) .
$$

Let the function $\varphi(a, b ; z)$ be given by:

$$
\varphi(a, b ; z)=z+\sum_{k=n+1}^{\infty} \frac{(a)_{k-1}}{(b)_{k-1}} z^{k},(b \neq 0,-1,-2,-3, \cdots),
$$

\footnotetext{
"Corresponding author.
}

where $(x)_{k}$ denotes the Pochhammer symbol (or the shifted factorial) defined by:

$$
(x)_{k}=\left\{\begin{array}{cl}
1 & \text { for } k=0, x \in \mathbb{C}-\{0\}, \\
x(x+1) \cdots(x+k-1) & \text { for } k \in \mathbb{N}=1,2,3, \cdots .
\end{array}\right.
$$

Carlson and Shaffer [1] introduced a convolution operator on $\mathcal{A}$ involving an incomplete beta function as:

$$
L(a, b) f(z):=\varphi(a, b ; z) * f(z) .
$$

Our work here motivated by Catas [2], who introduced an operator on $\mathcal{A}$ as follows:

$$
D_{l}^{m, \lambda} f(z)=z+\sum_{k=n+1}^{\infty}\left(\frac{1+\lambda(k-1)+l}{1+l}\right)^{m} a_{k} z^{k},
$$

where

$$
z \in \mathbb{U}, \lambda \geq 0, m \in \mathbb{Z}, l \geq 0 .
$$

Now, using the Hadamard product (or convolution), the authors (cf. [3,4]) introduced the following linear operator:

Definition 1.1 Let

$$
\phi_{l}^{m, \lambda}(a, b ; z)=\sum_{k=n+1}^{\infty}\left(\frac{1+\lambda(k-1)+l}{1+l}\right)^{m} \frac{(a)_{k-1}}{(b)_{k-1}} z^{k},
$$

where

$$
(z \in U, b \neq 0,-1,-2,-3, \cdots), \lambda \geq 0, m \in \mathbb{Z}, l \geq 0,
$$

and $(x)_{k}$ is the Pochhammer symbol. We defines $a$ linear operator $D_{l}^{m, \lambda}(a, b): \mathcal{A}(n) \rightarrow \mathcal{A}(n)$ by the following Hadamard product: 


$$
\begin{aligned}
& D_{l}^{m, \lambda}(a, b) f(z):=\phi_{l}^{m, \lambda}(a, b ; z) * f(z) \\
& =z+\sum_{k=n+1}^{\infty}\left(\frac{1+\lambda(k-1)+l}{1+l}\right)^{m} \frac{(a)_{k-1}}{(b)_{k-1}} a_{k} z^{k},
\end{aligned}
$$

where

$$
(z \in U, b \neq 0,-1,-2,-3, \cdots), \lambda \geq 0, m \in \mathbb{Z}, l \geq 0,
$$

and $(x)_{k}$ the Pochhammer symbol .

Special cases of this operator include:

- $D_{0}^{m, 0}(a, b) f(z)=D_{l}^{0, \lambda}(a, b) f(z)=L(a, b) f(z)$, see [1].

- the Catas drivative operator [2]: $D_{l}^{m, \lambda}(1,1) f(z)$.

- the Ruscheweyh derivative operator [5] in the cases:

$$
D_{0}^{0,0}(\beta+1,1) f(z)=D^{\beta} f(z) ; \beta \geq-1 .
$$

- the Salagean derivative operator [6]: $D_{0}^{m, 1}(1,1) f(z)$.

- the generalized Salagean derivative operator introduced by Al-Oboudi [7]: $D_{0}^{m, \lambda}(1,1) f(z)$.

- Note that:

$$
\begin{aligned}
& D_{0}^{0, \lambda}(a, b) f(z)=L(a, b) f(z), \\
& D_{l}^{1, \lambda}(a, b) f(z)=\left(\frac{1-\lambda+l}{1+l}\right) L(a, b) f(z) \\
& +\frac{\lambda z}{1+l}(L(a, b) f(z))=D_{\lambda}(L(a, b) f(z)), \lambda \geq 0 .
\end{aligned}
$$

Let $\mathcal{T}(n)$ denote the class of functions $f(z)$ of the form

$$
f(z)=z=\sum_{k=n+1}^{\infty} a_{k} z^{k}, a_{k} \geq 0, n \in \mathbb{N},
$$

which are analytic in the open unit disc.

Following the earlier investigations by [8] and [9], we define $(n, \eta)$-neighborhood of a function $f(z) \in \mathcal{T}(n)$ by

$$
\begin{aligned}
& N_{n, \eta}(f) \\
& =\left\{g(z)=z-\sum_{k=n+1}^{\infty} b_{k} z^{k} \in \mathcal{T}(n): \sum_{k=n+1}^{\infty} k\left|a_{k}-b_{k}\right| \leq \eta\right\}
\end{aligned}
$$

or,

$$
N_{n, \eta}(h):=\left\{g(z)=z-\sum_{k=n+1}^{\infty} b_{k} z^{k} \in \mathcal{T}(n): \sum_{k=n+1}^{\infty} k\left|b_{k}\right| \leq \eta\right\},
$$

where $h(z)=z$.

Let $\mathcal{S}_{n}^{*}(\alpha)$ denote the subclass of $\mathcal{T}(n)$ consisting of functions which satisfy

$$
\operatorname{Re}\left(\frac{z f^{\prime}(z)}{f(z)}\right)>\alpha, z \in U, 0 \leq \alpha<1 .
$$

A function $f(z)$ in $\mathcal{S}_{n}^{*}(\alpha)$ is said to be starlike of order $\alpha$ in $U$.
A function $f(z) \in \mathcal{T}(n)$ is said to be convex of order $\alpha$ it it satisfies

$$
\operatorname{Re}\left(1+\frac{z f^{\prime \prime}(z)}{f^{\prime}(z)}\right)>\alpha, z \in U, 0 \leq \alpha<1 .
$$

We denote by $\mathcal{C}_{n}(\alpha)$ the subclass of $\mathcal{T}(n)$ consisting of all such functions [10].

The unification of the classes $\mathcal{S}_{n}^{*}(\alpha)$ and $\mathcal{C}_{n}(\alpha)$ is provided by the class $\mathcal{T}_{n}(\alpha, \gamma)$ of functions

$f(z) \in \mathcal{T}(n)$ which also satisfy the following inequality

$$
\begin{aligned}
& \operatorname{Re}\left(\frac{z f^{\prime}(z)+\gamma z^{2} f^{\prime \prime}(z)}{\gamma z f^{\prime}(z)+(1-\gamma) f(z)}\right)>\alpha, \\
& z \in U, 0 \leq \alpha<1,0 \leq \gamma \leq 1 .
\end{aligned}
$$

The class $\mathcal{T}_{n}(\alpha, \gamma)$ was investigated by Altintas [11].

Now, by using $D_{l}^{m, \lambda}(a, b)$ we will define a new class of starlike functions.

Definition 1.2 Let

$$
\begin{aligned}
& 0 \leq \alpha<1,0 \leq \gamma \leq 1, \\
& b \neq 0,-1,-2,-3, \cdots, m \in \mathbb{Z}, l \geq 0, \lambda \geq 0 .
\end{aligned}
$$

A function $f$ belonging to $\mathcal{T}(n)$ is said to be in the class $\mathcal{T}_{\lambda, l}^{m}(n, \alpha, \gamma, a, b)$ if and only if

$\operatorname{Re}\left\{\frac{(1-\gamma) z\left(D_{l}^{m, \lambda}(a, b) f(z)\right)^{\prime}+\gamma z\left(D_{l}^{m+1, \lambda}(a, b) f(z)\right)^{\prime}}{(1-\gamma) z\left(D_{l}^{m, \lambda}(a, b) f(z)\right)+\gamma z\left(D_{l}^{m+1, \lambda}(a, b) f(z)\right)}\right\}$ $>\alpha, z \in U$.

Remark 1.3 The class $\mathcal{T}_{\lambda, l}^{m}(n, \alpha, \gamma, a, b)$ is a generalization of the following subclasses:

i) $\mathcal{T}_{1,0}^{0}(1, \alpha, 0,1,1) \equiv \mathcal{T}^{*}(\alpha) \equiv \mathcal{S}_{1}^{*}(\alpha)$ and

$\mathcal{T}_{1,0}^{1}(1, \alpha, 0,1,1) \equiv \mathcal{C}(\alpha) \equiv \mathcal{C}_{1}(\alpha)$ defined and studied by [12];

ii) $\mathcal{T}_{1,0}^{0}(1, \alpha, 0,1,1)$ and $\mathcal{T}_{1,0}^{1}(1, \alpha, 0,1,1)$ studied by [13] and [14];

iii) $\mathcal{T}_{1,0}^{m}(1, \alpha, 0,1,1) \equiv \mathcal{T}(m, \alpha)$ studied by [15];

iv) $\mathcal{T}_{1,0}^{0}(n, \alpha, \gamma, 1,1)$ studied by [16].

Now, we shall use the same method by [17] to establish certain coefficient estimates relating to the new introduced class.

\section{Coefficient Estimates}

Theorem 2.1 Let the function $f$ be defined by (1). Then $f$ belongs to the class $\mathcal{T}_{\lambda, l}^{m}(n, \alpha, \gamma, a, b)$ if and only if

$$
\begin{aligned}
& \sum_{k=n+1}^{\infty} c_{k}(m, \lambda, l, a, b)(k-\alpha)[1+l+\gamma \lambda(k-1)] a_{k} \\
& \leq(1+l)(1-\alpha),
\end{aligned}
$$

where 


$$
\begin{aligned}
& c_{k}(m, \lambda, l, a, b) \\
& =\left[\frac{1+\lambda(k-1)+l}{1+l}\right]^{m} \frac{(a)_{k-1}}{(b)_{k-1}} .
\end{aligned}
$$

The result is sharp and the extremal functions are

$$
f_{k}(z)=z-\frac{(1+l)(1-\alpha)}{c_{k}(m, \lambda, l, a, b)(k-\alpha)[1+l+\gamma \lambda(k-1)]} z^{k} \text {, (9) }
$$
$k \geq n+1$.

Proof: Assume that the inequality (7) holds and let $|z|=1$. Then we have

$$
\begin{aligned}
& \left|\frac{(1-\gamma) z\left(D_{l}^{m, \lambda}(a, b) f(z)\right)^{\prime}+\gamma z\left(D_{l}^{m+1, \lambda}(a, b) f(z)\right)^{\prime}}{(1-\gamma) z\left(D_{l}^{m, \lambda}(a, b) f(z)\right)+\gamma z\left(D_{l}^{m+1, \lambda}(a, b) f(z)\right)}-1\right| \\
& =\left|\frac{\sum_{k=n+1}^{\infty}\left[\frac{1+\lambda(k-1)+l}{1+l}\right]^{m}\left[\frac{1+l+\gamma \lambda(k-1)}{1+l}\right] \frac{(a)_{k-1}}{(b)_{k-1}}(k-1) a_{k} z^{k-1} \mid}{1-\sum_{k=n+1}^{\infty}\left[\frac{1+\lambda(k-1)+l}{1+l}\right]^{m}\left[\frac{1+l+\gamma \lambda(k-1)}{1+l}\right] \frac{(a)_{k-1}}{(b)_{k-1}} a_{k} z^{k-1}}\right| \\
& \leq 1+\frac{\sum_{k=n+1}^{\infty}\left[\frac{1+\lambda(k-1)+l}{1+l}\right]^{m}\left[\frac{1+l+\gamma \lambda(k-1)}{1+l}\right] \frac{(a)_{k-1}}{(b)_{k-1}} k a_{k}-1}{1-\sum_{k=n+1}^{\infty}\left[\frac{1+\lambda(k-1)+l}{1+l}\right]^{m}\left[\frac{1+l+\gamma \lambda(k-1)}{1+l}\right] \frac{(a)_{k-1}}{(b)_{k-1}} a_{k}} .
\end{aligned}
$$

Consequently, by the maximum modulus theorem one obtains

$$
\begin{array}{r}
f(z) \in \mathcal{T}_{\lambda, l}^{m}(n, \alpha, \gamma, a, b) . \quad \text { Then from (6) we find that } \\
\operatorname{Re}\left\{\frac{z-\sum_{k=n+1}^{\infty}\left[\frac{1+\lambda(k-1)+l}{1+l}\right]^{m}\left[\frac{1+l+\gamma \lambda(k-1)}{1+l}\right] \frac{(a)_{k-1}}{(b)_{k-1}} k a_{k} z^{k}}{z-\sum_{k=n+1}^{\infty}\left[\frac{1+\lambda(k-1)+l}{1+l}\right]^{m}\left[\frac{1+l+\gamma \lambda(k-1)}{1+l}\right] \frac{(a)_{k-1}}{(b)_{k-1}} a_{k} z^{k}}\right\} \alpha .
\end{array}
$$

Choose values of $\mathrm{Z}$ on the real axis such that

$$
\frac{(1-\gamma) z\left(D_{l}^{m, \lambda}(a, b) f(z)\right)^{\prime}+\gamma z\left(D_{l}^{m+1, \lambda}(a, b) f(z)\right)^{\prime}}{(1-\gamma) z\left(D_{l}^{m, \lambda}(a, b) f(z)\right)+\gamma z\left(D_{l}^{m+1, \lambda}(a, b) f(z)\right)}
$$

is real. Letting $z \rightarrow 1^{-}$through real values, we obtain

or, equivalently

$$
\operatorname{Re}\left\{\frac{1-\sum_{k=n+1}^{\infty}\left[\frac{1+\lambda(k-1)+l}{1+l}\right]^{m}\left[\frac{1+l+\gamma \lambda(k-1)}{1+l}\right] \frac{(a)_{k-1}}{(b)_{k-1}} k a_{k}}{1-\sum_{k=n+1}^{\infty}\left[\frac{1+\lambda(k-1)+l}{1+l}\right]^{m}\left[\frac{1+l+\gamma \lambda(k-1)}{1+l}\right] \frac{(a)_{k-1}}{(b)_{k-1}} a_{k}}\right\} \geq \alpha,
$$

$$
\begin{gathered}
1-\sum_{k=n+1}^{\infty}\left[\frac{1+\lambda(k-1)+l}{1+l}\right]^{m}\left[\frac{1+l+\gamma \lambda(k-1)}{1+l}\right] \frac{(a)_{k-1}}{(b)_{k-1}} k a_{k} \\
\geq \alpha\left\{1-\sum_{k=n+1}^{\infty}\left[\frac{1+\lambda(k-1)+l}{1+l}\right]^{m}\left[\frac{1+l+\gamma \lambda(k-1)}{1+l}\right] \frac{(a)_{k-1}}{(b)_{k-1}} a_{k}\right\},
\end{gathered}
$$


which gives (7).

Remark 2.2 In the special case $a=b=1$, Theorem 2.1 yields a result given earlier by [17].

Remark 2.3 In the special case $\lambda=1, l=0$, $a=b=1$, Theorem 2.2 yields a result given earlier by [6].

Theorem 2.4 Let the function $f$ defined by (3) be in the class $\mathcal{T}_{\lambda, l}^{m}(n, \alpha, \gamma, a, b)$. Then

$$
\sum_{k=n+1}^{\infty} a_{k} \leq \frac{(1+l)(1-\alpha)}{c_{n+1}(m, \lambda, l, a, b)(1+l+\gamma \lambda n)(n+1-\alpha)},
$$

and

$$
\sum_{k=n+1}^{\infty} k a_{k} \leq \frac{(1+l)(1-\alpha)(n+1)}{c_{n+1}(m, \lambda, l, a, b)(1+l+\gamma \lambda n)(n+1-\alpha)} .
$$

The equality in (10) and (11) is attained for the function $f$ given by (9).

Proof: By using Theorem 2.2, we find from (6) that

$$
\begin{aligned}
& (1+l+\gamma \lambda n)(n+1-\alpha) c_{n+1}(m, \lambda, l, a, b) \sum_{k=n+1}^{\infty} a_{k} \\
& \leq \sum_{k=n+1}^{\infty} c_{k}(m, \lambda, l, a, b)(k-\alpha)[1+l+\gamma \lambda(k-1)] a_{k} \\
& \leq(1+l)(1-\alpha),
\end{aligned}
$$

which immediately yields the first assertion (10) of Theorem 2.3.

On the other hand, taking into account the inequality (6), we also have

$$
\begin{aligned}
& (1+l+\gamma \lambda n) c_{n+1}(m, \lambda, l, a, b) \sum_{k=n+1}^{\infty}(k-\alpha) a_{k} \\
& \leq(1+l)(1-\alpha),
\end{aligned}
$$

that is

$$
\begin{aligned}
& (1+l+\gamma \lambda n) c_{n+1}(m, \lambda, l, a, b) \sum_{k=n+1}^{\infty} k a_{k} \\
& \leq(1+l)(1-\alpha)+\alpha(1+l+\gamma \lambda n) c_{n+1}(m, \lambda, l, a, b) \sum_{k=n+1}^{\infty} a_{k},
\end{aligned}
$$

which, in view of the coefficient inequality (10), can be put in the form

$$
\begin{aligned}
& (1+l+\gamma \lambda n) c_{n+1}(m, \lambda, l, a, b) \sum_{k=n+1}^{\infty} k a_{k} \\
& \leq(1+l)(1-\alpha)+\alpha(1+l+\gamma \lambda n) c_{n+1}(m, \lambda, l, a, b) \\
& \frac{(1+l)(1-\alpha)}{c_{n+1}(m, \lambda, l, a, b)(1+l+\gamma \lambda n)(n+1-\alpha)},
\end{aligned}
$$

and this completes the proof of (11).

\section{Closure Theorem}

Theorem 3.1 Let the function $f_{j}(z),(j=1,2, \cdots, m)$ be defined by

$$
f_{j}(z)=z-\sum_{k=n+1}^{\infty} a_{k j} z^{k},
$$

for $z \in U$, be in the class $\mathcal{T}_{\lambda, l}^{m}(n, \alpha, \gamma, a, b)$ then the function $h(z)$ defined by

$$
h(z)=z-\sum_{k=n+1}^{\infty} b_{k} z^{k},\left(a_{k j}>0\right),
$$

also belongs to the class $\mathcal{T}_{\lambda, l}^{m}(n, \alpha, \gamma, a, b)$, where

$$
b_{k}=\frac{1}{m} \sum_{j=n}^{m} a_{k j} .
$$

Proof: Since $f_{j}(z) \in \mathcal{T}_{\lambda, l}^{m}(n, \alpha, \gamma, a, b)$, it follows from Theorem 2.1, that

$$
\begin{aligned}
& \sum_{k=n+1}^{\infty} c_{k}(m, \lambda, l, a, b)(k-\alpha)[1+l+\gamma \lambda(k-1)] a_{k j} \\
& <(1+l)(1-\alpha),(j=1,2, \cdots, m) .
\end{aligned}
$$

Therefore,

$$
\begin{aligned}
& \sum_{k=n+1}^{\infty} c_{k}(m, \lambda, l, a, b)(k-\alpha)[1+l+\gamma \lambda(k-1)] b_{k} \\
= & \sum_{k=n+1}^{\infty} c_{k}(m, \lambda, l, a, b)(k-\alpha)[1+l+\gamma \lambda(k-1)]\left(\frac{1}{m} \sum_{j=n}^{m} a_{k j}\right) \\
= & \frac{1}{m} \sum_{j=n}^{m}\left(\sum_{k=n+1}^{\infty} c_{k}(m, \lambda, l, a, b)(k-\alpha)[1+l+\gamma \lambda(k-1)] a_{k j}\right) \\
< & (1+l)(1-\alpha) .
\end{aligned}
$$

Hence by Theorem 2.1, $h(z) \in \mathcal{T}_{\lambda, l}^{m}(n, \alpha, \gamma, a, b)$ also.

Morever, we shall use the same method by [17] to prove the distrotion Theorems.

\section{Distortion Theorems}

Theorem 4.1 Let the function $f$ defined by (1) be in the class $\mathcal{T}_{\lambda, l}^{m}(n, \alpha, \gamma, a, b)$. Then we have

$$
\begin{aligned}
& \left|D_{l}^{i, \lambda}(a, b) f(z)\right| \\
& \geq|z|-\frac{(1+l)(1-\alpha)}{c_{k}(m-i, \lambda, l, a, b)(n+1-\alpha)(1+l+\gamma \lambda n)}|z|^{n+1},
\end{aligned}
$$

and

$$
\begin{aligned}
& \left|D_{l}^{i, \lambda}(a, b) f(z)\right| \\
& \leq|z|+\frac{(1+l)(1-\alpha)}{c_{k}(m-i, \lambda, l, a, b)(n+1-\alpha)(1+l+\gamma \lambda n)}|z|^{n+1},
\end{aligned}
$$

for $z \in U$, where $0 \leq i \leq m$ and $c_{k}(m-i, \lambda, l, a, b)$ is given by (8).

The equalities in (12) and (13) are attained for the function $f$ given by

$$
\begin{aligned}
& f_{n+1}(z) \\
& =z-\frac{(1-\alpha)(1+l)^{m+1}}{(1+\lambda n+l)^{m}(n+1-\alpha)(1+l+\gamma \lambda n)} z^{n+1} .
\end{aligned}
$$


Proof: Note that $f \in \mathcal{T}_{\lambda, l}^{m}(n, \alpha, \gamma, a, b)$ if and only if $D_{l}^{i, \lambda}(a, b) f(z) \in \mathcal{T}_{\lambda, l}^{m-i}(n, \alpha, \gamma, a, b)$, where

$$
D_{l}^{i, \lambda}(a, b) f(z)=z-\sum_{k=n+1}^{\infty} c_{k}(i, \lambda, l, a, b) a_{k} z^{k} .
$$

By Theorem 2.2, we know that

$$
\begin{aligned}
& c_{k}(m-i, \lambda, l, a, b)(n+1-\alpha) \\
& \cdot(1+l+\gamma \lambda n) \sum_{k=n+1}^{\infty} c_{k}(i, \lambda, l, a, b) a_{k} \\
& \leq \sum_{k=n+1}^{\infty} c_{k}(m, \lambda, l, a, b)(k-\alpha)[1+l+\gamma \lambda(k-1)] a_{k} \\
& \leq(1+l)(1-\alpha),
\end{aligned}
$$

that is

$$
\begin{aligned}
& \sum_{k=n+1}^{\infty} c_{k}(i, \lambda, l, a, b) a_{k} \\
& \leq \frac{(1+l)(1-\alpha)}{c_{k}(m-i, \lambda, l, a, b)(n+1-\alpha)(1+l+\gamma \lambda n)} .
\end{aligned}
$$

The assertions of (12) and (13) of Theorem 4.1 follow immediately. Finally, we note that the equalities (12) and (13) are attained for the function $f$ defined by

$$
\begin{aligned}
& D_{l}^{i, \lambda}(a, b) f(z) \\
& =z-\frac{(1+l)(1-\alpha)}{c_{k}(m-i, \lambda, l, a, b)(n+1-\alpha)(1+l+\gamma \lambda n)} z^{n+1} .
\end{aligned}
$$

This completes the proof of Theorem 4.1.

Remark 4.2 In the special case $a=b=1$, Theorem 4.1 yields a result given earlier by [17].

Corollary 4.3 Let the function $f$ defined by (1) be in the class $\mathcal{T}_{\lambda, l}^{m}(n, \alpha, \gamma, a, b)$. Then we have

$$
\begin{aligned}
& |f(z)| \\
& \geq|z|-\frac{(1+l)(1-\alpha)}{c_{k}(m, \lambda, l, a, b)(n+1-\alpha)(1+l+\gamma \lambda n)}|z|^{n+1},
\end{aligned}
$$

and

$$
\begin{aligned}
& |f(z)| \\
& \leq|z|+\frac{(1+l)(1-\alpha)}{c_{k}(m, \lambda, l, a, b)(n+1-\alpha)(1+l+\gamma \lambda n)}|z|^{n+1},
\end{aligned}
$$

for $z \in U$. The equalities in (15) and (16) are attained for the function $f_{n+1}$ given in (14).

Corollary 4.4 Let the function $f$ defined by (1) be in the class $\mathcal{T}_{\lambda, l}^{m}(n, \alpha, \gamma, a, b)$. Then we have

$$
\begin{aligned}
& \left|f^{\prime}(z)\right| \\
& \geq 1-\frac{(1+l)(1-\alpha)(n+1)}{c_{k}(m, \lambda, l, a, b)(n+1-\alpha)(1+l+\gamma \lambda n)}|z|^{n},
\end{aligned}
$$

and

$$
\begin{aligned}
& \left|f^{\prime}(z)\right| \\
& \leq 1+\frac{(1+l)(1-\alpha)(n+1)}{c_{k}(m, \lambda, l, a, b)(n+1-\alpha)(1+l+\gamma \lambda n)}|z|^{n},
\end{aligned}
$$

for $z \in U$. The equalities in (17) and (18) are attained for the function $f_{n+1}$ given in (14).

Corollary 4.5 Let the function $f$ defined by (3) be in the class $\mathcal{T}_{\lambda, l}^{m}(n, \alpha, \gamma, a, b)$. Then the unit disc is mapped onto a domain that contains the disc

$$
\begin{aligned}
& |w| \\
& <\frac{c_{k}(m, \lambda, l, a, b)(n+1-\alpha)(1+l+\gamma \lambda n)-(1+l)(1-\alpha)}{c_{k}(m, \lambda, l, a, b)(n+1-\alpha)(1+l+\gamma \lambda n)} .
\end{aligned}
$$

The result is sharp with the extremal function $f_{n+1}$ given in (14).

\section{Integral Operators}

Theorem 5.1 Let the function $f(z)$ defined by (1) be in the class $\mathcal{T}_{\lambda, l}^{m}(n, \alpha, \gamma, a, b)$ and let $c$ be a real number such that $c>-1$. Then $F(z)$, defined by

$$
F(z)=\frac{c+1}{z^{c}} \int_{0}^{z} t^{c-1} f(t) \mathrm{d} t,
$$

also belongs to the class $\mathcal{T}_{\lambda, l}^{m}(n, \alpha, \gamma, a, b)$

Proof: From the representation of $F(z)$, it is obtained that

$$
F(z)=z-\sum_{k=n+1}^{\infty} b_{k} z^{k},(n \in \mathbb{N}),
$$

where

Therefore

$$
b_{k}=\left(\frac{c+1}{k+c}\right) a^{k}
$$

$$
\begin{aligned}
& \sum_{k=n+1}^{\infty} c_{k}(m, \lambda, l, a, b)(k-\alpha)[1+l+\gamma \lambda(k-1)] b_{k} \\
& \cdot \sum_{k=n+1}^{\infty} c_{k}(m, \lambda, l, a, b)(k-\alpha)[1+l+\gamma \lambda(k-1)]\left(\frac{c+1}{k+c}\right) a^{k} \\
& \leq \sum_{k=n+1}^{\infty} c_{k}(m, \lambda, l, a, b)(k-\alpha)[1+l+\gamma \lambda(k-1)] a^{k} \\
& \leq(1+l)(1-\alpha),
\end{aligned}
$$

since $f(z)$ belongs to $\mathcal{T}_{\lambda, l}^{m}(n, \alpha, \gamma, a, b)$ so by virtue of Theorem 2.1, $F(z)$ is also element of

$\mathcal{T}_{\lambda, l}^{m}(n, \alpha, \gamma, a, b)$

\section{Acknowledgements}

The work presented here was partially supported by LRGS/TD/2011/UKM/ICT/03/02. 


\section{REFERENCES}

[1] B. C. Carlson and D. B. Shaffer, "Starlike and Prestarlike Hypergeometric Functions," SIAM Journal on Mathematical Analysis, Vol. 15, No. 4, 1984, pp. 737-745. doi:10.1137/0515057

[2] A. Catas, "On a Certain Differential Sandwich Theorem Associated with a New Generalized Derivative Operator," General Mathematics, Vol. 17, No. 4, 2009, pp. 83-95.

[3] A. A. Amer and M. Darus, "A Distortion Theorem for a Certain Class of Bazilevic Function,” International Journal of Mathematical Analysis, Vol. 6, No. 12, 2012, pp. 591-597.

[4] A. A. Amer and M. Darus, "On a Property of a Subclass of Bazilevic Functions,” Missouri Journal of Mathematical Sciences, In Press.

[5] St. Ruscheweyh, "New Criteria for Univalent Functions," Proceedings of the American Mathematical Society, Vol. 49, No. 1, 1975, pp. 109-115.

[6] G. S. Salagean, "Subclasses of Univalent Functions," Lecture Notes in Mathematics, Vol. 1013, 1983, pp. 362372. doi:10.1007/BFb0066543

[7] F. M. Al-Oboudi, "On Univalent Functions Defined by a Generalized Salagean Operator,” International Journal of Mathematics and Mathematical Sciences, Vol. 2004, No. 27, 2004, pp. 1429-1436. doi:10.1155/S0161171204108090

[8] A. W. Goodman, "Univalent Functions and Nonanalytic Curves," Proceedings of the American Mathematical Society, Vol. 8, No. 3, 1957, pp. 598-601. doi:10.1090/S0002-9939-1957-0086879-9

[9] St. Ruscheweyh, "Neighborhoods of Univalent Functions," Proceedings of the American Mathematical Society, Vol.
81, No. 4, 1981, pp. 521-527. doi:10.1090/S0002-9939-1981-0601721-6

[10] O. Alintas, H. Irmak and H. M. Srivastava, "Fractional Calculus and Certain Starlike Functions with Negative Coefficietns," Computers \& Mathematics with Applications, Vol. 30, No. 2, 1995, pp. 9-15. doi:10.1016/0898-1221(95)00073-8

[11] O. Alintas, "On a Subclass of Certain Starlike Functions with Negative Coefficients,” Journal of the Mathematical Society of Japan, Vol. 36, 1991, pp. 489-495.

[12] H. Silverman, "Univalent Functions with Negative Coefficients," Proceedings of the American Mathematical Society, Vol. 51, No. 1, 1975, pp. 109-116. doi:10.1090/S0002-9939-1975-0369678-0

[13] S. K. Chatterjea, “On Starlike Functions,” Journal of Pure Mathematics, Vol. 1, 1981, pp. 23-26.

[14] H. M. Srivastava, S. Owa and S. K. Chatterjea, "A Note on Certain Classes of Starlike Functions," Rendiconti del Seminario Matematico della Università di Padova, Vol. 77, 1987, pp.115-124.

[15] M. D. Hur and G. H. Oh, "On Certain Class of Analytic Functions with Negative Coefficients,” Pusan Kyongnam Mathematical Journal, Vol. 5, No. 1, 1989, pp. 69-80.

[16] M. Kamali, "Neighborhoods of a New Class of p-Valently Functions with Negative Coefficients," Mathematical Inequalities \& Applications, Vol. 9, No. 4, 2006, pp. 661670. doi:10.7153/mia-09-59

[17] A. Catas, "Neighborhoods of a Certain Class of Analytic Functions with Negative Coefficients,” Banach Journal of Mathematical Analysis, Vol. 3, No. 1, 2009, pp. 111121. 